\title{
Anemia em crianças e mulheres indígenas no Brasil: revisão sistemática
}

\author{
Anemia in indigenous women and children in Brazil: \\ a systematic review
}

\author{
Juliana Souza Andrade Lício ${ }^{1}$ \\ Thatiana Regina Fávaro ${ }^{2}$ \\ Célia Regina Moutinho de Miranda Chaves ${ }^{1}$
}

${ }^{1}$ Instituto Fernandes Figueira, Fiocruz. Av. Rui Barbosa 716, Flamengo. 22250-020 Rio de Janeiro RJ Brasil.

liciojuliana@yahoo.com.br

${ }^{2}$ Faculdade de Nutrição,

Universidade Federal de

Alagoas. Maceió AL Brasil.

\begin{abstract}
Iron-deficiency anemia can be considered the most important nutritional deficiency in Brazil. The scope of this article was to conduct a systematic review of the literature in order to describe the frequency of anemia in indigenous women and children in Brazil, and gather information on associated factors. A search of the PubMed, Scopus and Lilacs databases was conducted. Seventeen studies were selected. The rates of prevalence of anemia ranged from $23.1 \%$ $74.6 \% ; 42.9 \%-92.3 \%$ and $29.6 \%-84 \%$, for children aged 6 to 119 months, 6 to 23 months and 6 to 59 months, respectively. Two studies were conducted with women of childbearing age and rates of $67 \%$ were found among the Surui and $16.1 \%$ among the Xukuru of Ororubá. The majority of authors attribute the prevalence of anemia to inadequate sanitary conditions, diets deficient in iron and essential micronutrients and a lack of access to basic health services. The findings highlight the importance of anemia among the indigenous peoples studied, which has been reflected by a substantial increase in research over the last twenty years.
\end{abstract}

Key words Iron-deficiency anemia, Iron deficiency, Health of indigenous peoples
Resumo A anemia ferropriva pode ser considerada a mais importante carência nutricional no Brasil. O objetivo deste artigo foi realizar uma revisão sistemática da literatura sobre anemia em mulheres e crianças indigena no Brasil, com vistas a reunir informações relevantes sobre os fatores associados, assim como a frequência do agravo nesta população. Realizou-se busca nas bases bibliográficas Pubmed, Scopus e Lilacs, além do Portal de Teses em Saúde Pública da BVS. Foram selecionados 17 estudos. As prevalências de anemia encontradas variaram de 23,1\% a 74,6\%; 42,9\% a 92,3\% e de 29,6\% a 84\% para crianças na idade seis a 119 meses, de seis a 23 meses e de seis a 59 meses, respectivamente. Os dois estudos que foram realizados especificamente com mulheres em idade fértil encontraram taxas de 67\% nas Suruí e de 16,1\% entre as Xukuru do Ororubá. A maioria dos autores relaciona a maior prevalência da anemia às condições sanitárias impróprias, às dietas insuficientes em ferro e micronutrientes essenciais e à falta de acesso aos serviços básicos de saúde. Houve um perceptivel aumento substancial das pesquisas ao longo dos últimos vinte anos, cujos achados evidenciam a importância de se resolver o problema da anemia entre os povos indígenas investigados.

Palavras-chave Anemia ferropriva, Deficiência de ferro, Saúde de populações indígenas 


\section{Introdução}

A anemia ferropriva é considerada a carência nutricional mais prevalente no mundo, principalmente nos países em desenvolvimento ${ }^{1}$. Estimase que existam no mundo 1,62 bilhões de pessoas anêmicas, com maior prevalência nas crianças menores de cinco anos e o maior número absoluto de casos entre mulheres grávidas².

Os estudos realizados no Brasil, reunidos em recentes revisões de literatura mostraram que a anemia ferropriva pode ser considerada a mais importante carência nutricional do país, com prevalências sempre superiores em populações em evidente condição de iniquidade social ${ }^{3,4}$. Nas crianças e adolescentes a anemia interfere no aproveitamento escolar, no desenvolvimento psicomotor, no crescimento e na imunidade celular, enquanto que em adultos está associada principalmente à queda de produtividade, sendo as mulheres em idade reprodutiva as mais vulneráveis ${ }^{5}$. A ocorrência de anemia durante a gestação representa aumento de risco de mortalidade materna e perinatal ${ }^{3}$.

Apesar da grande deficiência no monitoramento da anemia em povos indígenas no cenário mundial, estudos relatam que as prevalências são maiores do que as observadas nas populações não indígenas, sendo relacionadas às condições inadequadas de vida, dieta insuficiente em ferro e outros micronutrientes essenciais e elevadas taxas de doenças infecciosas ${ }^{6}$. A situação de saúde frequentemente relatada para os povos indígenas no Brasil é de extrema precariedade, com elevadas taxas de morbimortalidade por doenças infecciosas, parasitárias e carenciais, entre as quais se destacam elevadas prevalências de desnutrição e de anemia no público materno infantil e a presença de excesso de peso na população adulta ${ }^{7-9}$. $\mathrm{O}$ único estudo de âmbito nacional com povos indígenas já realizado no país, o I Inquérito $\mathrm{Na}$ cional de Saúde e Nutrição dos Povos Indígenas no Brasil (doravante denominado "Inquérito Indígena"), realizado em 2008-2009, confirmou a anemia como relevante agravo no cenário epidemiológico para crianças e mulheres indígenas ${ }^{9}$.

Conhecer as especificidades dos fatores que determinam a presença e a magnitude da anemia em mulheres e crianças indígenas pode contribuir para o enfrentamento do problema com vistas à melhoria da saúde e da nutrição desta população. Neste contexto, o objetivo do corrente estudo foi realizar uma revisão sistemática da literatura da anemia em mulheres e crianças indígenas no Brasil, com vistas a reunir informa- ções relevantes sobre os fatores associados, assim como a frequência do agravo nesta população.

\section{Métodos}

Em julho de 2014 foi realizada uma busca eletrônica nas bases de dados PubMed da U.S. National Library of Medicine (http://www.ncbi.nlm.nih. gov/pubmed/), Literatura Latino-Americana e do Caribe em Ciências da Saúde - Lilacs (http://www. http://lilacs.bvsalud.org/) e Scopus (ww.scopus. com). Foram utilizados os seguintes descritores: anemia, hemoglobin, Iron Deficiency, indigenous population, health indigenous peoples, indigenous, south american indians, indigenous people, health indigenous peoples, indians, indigenous health, Brazil e seus correspondentes em português. Os levantamentos foram concluídos utilizando-se equações construídas a partir dos descritores e de operadores booleanos (AND e OR). Não foram feitas restrições quanto ao idioma e ao período do estudo. A fim de identificar resultados de estudos inéditos e ainda não publicados na forma de artigos científicos, foi consultado o Portal de Teses em Saúde Pública da Biblioteca Virtual em Saúde (BVS) (http://bases.bireme.br/cgi-bin/ wxislind.exe/iah/bvsSP/) onde foram utilizados os descritores indígena OR índio no campo "palavras", anemia OR estado nutricional no campo "descritor de assuntos". Além da busca eletrônica, também foram revisadas listas de referências dos artigos, dissertações e teses localizados, com o objetivo de identificar possíveis trabalhos relevantes não encontrados na busca eletrônica.

Os estudos localizados foram analisados de forma independente por duas das autoras e, com base nos resumos, foram selecionadas de acordo com o critério de inclusão: estudos originais que tiveram como objetivo investigar a prevalência de anemia ferropriva e/ou fatores associados em crianças e mulheres indígenas no Brasil. No caso da seleção de resumo de dissertações ou teses que tivessem seus resultados também publicados na forma de artigo, optou-se por selecionar somente os artigos.

Os resumos selecionados pelos critérios descritos tiveram seus textos lidos de forma integral enquanto os estudos que atendiam a algum dos critérios de exclusão foram eliminados. Tais critérios foram: a) identificação da anemia por método diferente da análise de hemoglobina e/ ou ferro sérico e não dosados em análise laboratorial ou por hemoglobinômetro portátil; b) ausência da identificação precisa do local do estudo; 
c) não especificação do pertencimento étnico; d) artigos de revisão. Em casos de duplicatas (dois ou mais estudos com a mesma amostra), o mais recente ou a publicação mais completa foi a utilizada e as demais, excluídas. Artigos identificados em mais de uma base de dados foram computados em apenas uma.

Os manuscritos selecionados por ambos os autores foram incluídos como parte do estudo e diante da ausência de concordância sobre a inclusão ou exclusão de alguma publicação, o terceiro autor foi consultado.

Para a extração dos dados das publicações selecionadas foi utilizado um formulário padronizado e pré-testado com quatro publicações não incluídas na revisão e que continham os seguintes campos: autores, ano da publicação, tipo de publicação, idioma, localização geográfica, pertencimento étnico, desenho do estudo, delineamento amostra, número de pessoas avaliadas, faixa etária, sexo, recursos e critérios diagnósticos, prevalência de anemia e fatores associados e outras análises realizadas no estudo. A extração dos dados foi realizada por um revisor e, posteriormente, conferida por um segundo avaliador. As diferenças na extração dos dados e as dúvidas novamente foram solucionadas em consenso analisando-se o artigo original. As publicações incluídas foram organizadas em tabelas, seguindo a ordem do ano de publicação do estudo.

\section{Resultados}

A estratégia de busca recuperou 126 publicações, sendo 112 artigos, 12 dissertações e 2 teses. Após a análise dos resumos de acordo com os critérios de inclusão restaram 24 publicações. A leitura dos textos completos resultou na permanência de 17 publicações, que tiveram seus resultados sistematizados. Por meio da consulta as listas das referências, foram identificadas três dissertações. A Figura 1 apresenta o fluxograma do processo de seleção das publicações. O resultado final da seleção sistemática incluiu 10 artigos originais publicados em revistas indexadas, 6 dissertações e uma tese. Os artigos de Horta et al. ${ }^{7}$ e Leite et al. ${ }^{9}$, referentes aos resultados do Inquérito Indígena não foram selecionados para comporem a presente revisão pela não especificação do pertencimento étnico, uma vez que se tratou de um inquérito de representatividade nacional de acordo com as macrorregiões do Brasil. No entanto, seus resultados foram amplamente utilizados ao longo da discussão do presente artigo.
Na Tabela 1 estão as características gerais dos estudos incluídos na revisão. Todos os estudos selecionados apresentavam desenho transversal e apenas Leite ${ }^{10}$ e Fávaro ${ }^{11}$ realizaram investigações por meio de amostragem populacional, sendo que os demais buscaram incluir o maior número de indivíduos possível. A maioria dos estudos foi realizada nas regiões Norte e Centro-Oeste do país e concentradas em localidades nos limites da Amazônia Legal. Apenas o estudo de Carvalho-Costa et al. ${ }^{12}$ foi realizado entre indígenas não aldeados, moradores da área urbana da cidade Santa Izabel do Rio Negro (AM). Com exceção do artigo de Carvalho-Costa et al. ${ }^{12}$ publicado em língua inglesa, os demais foram publicados em português.

$\mathrm{O}$ recurso diagnóstico mais utilizado foi a dosagem de hemoglobina, por meio do hemoglobinômetro portátil HemoCue. Os critérios de diagnóstico da anemia foram baseados principalmente em recomendações da Organização Mundial de Saúde dos anos 1975 e 2001 e com pontos de corte similares para cada segmento.

O número de indivíduos avaliados variou de $49^{13}$ a $1069^{11}$, considerando todas as faixas de idade. Seis estudos ${ }^{10,11,14-16}$ apresentaram resultados referentes a mulheres indígenas e, com exceção de Koury Filho ${ }^{15}$ e de Orellana et al. ${ }^{17}$, todos avaliaram crianças. Koury Filho ${ }^{15}$ não especificou a faixa etária das mulheres indígenas analisadas. Ao todo as publicações encontradas investigaram 10 etnias distintas, sendo que quatro foram investigadas mais de uma vez. Sobre os Suruí ${ }^{17-19} \mathrm{e}$ os Xavante ${ }^{10,14,16}$ foram publicados três estudos e com os povos Teréna ${ }^{13,20}$ e Guarani ${ }^{21,22}$ foram encontrados dois estudos.

Considerando crianças de seis a 59 meses a prevalência de anemia variou de $29,6 \%$ a $92,3 \%$. As prevalências variaram de $23,1 \%$ a $74,6 \%$ naqueles que investigaram crianças na idade seis a 119 meses. As menores prevalências de anemia, tanto em crianças quanto em mulheres em idade fértil, foram encontradas entre a população indígena Xukuru do Ororubá, do Nordeste brasileiro ${ }^{11}$. Orellana et al. ${ }^{17}$ que também estudou mulheres em idade fértil, encontrando prevalências consideravelmente superiores. A prevalência de anemia em mulheres Xukuru, não gestantes, de 14 a 49 anos, foi 19,9\%, e em mulheres Suruí de 15 a 49 anos, 67\% ${ }^{11}$ (Tabela 2).

No primeiro estudo realizado entre as crianças Suruí ${ }^{18}$ a prevalência de anemia entre as menores de nove anos foi de 71,2\%, valor inferior ao observado no estudo de Orellana et al. ${ }^{19}$, de $80,5 \%$ e realizado cerca de 20 anos depois, também com crianças desse grupo. 


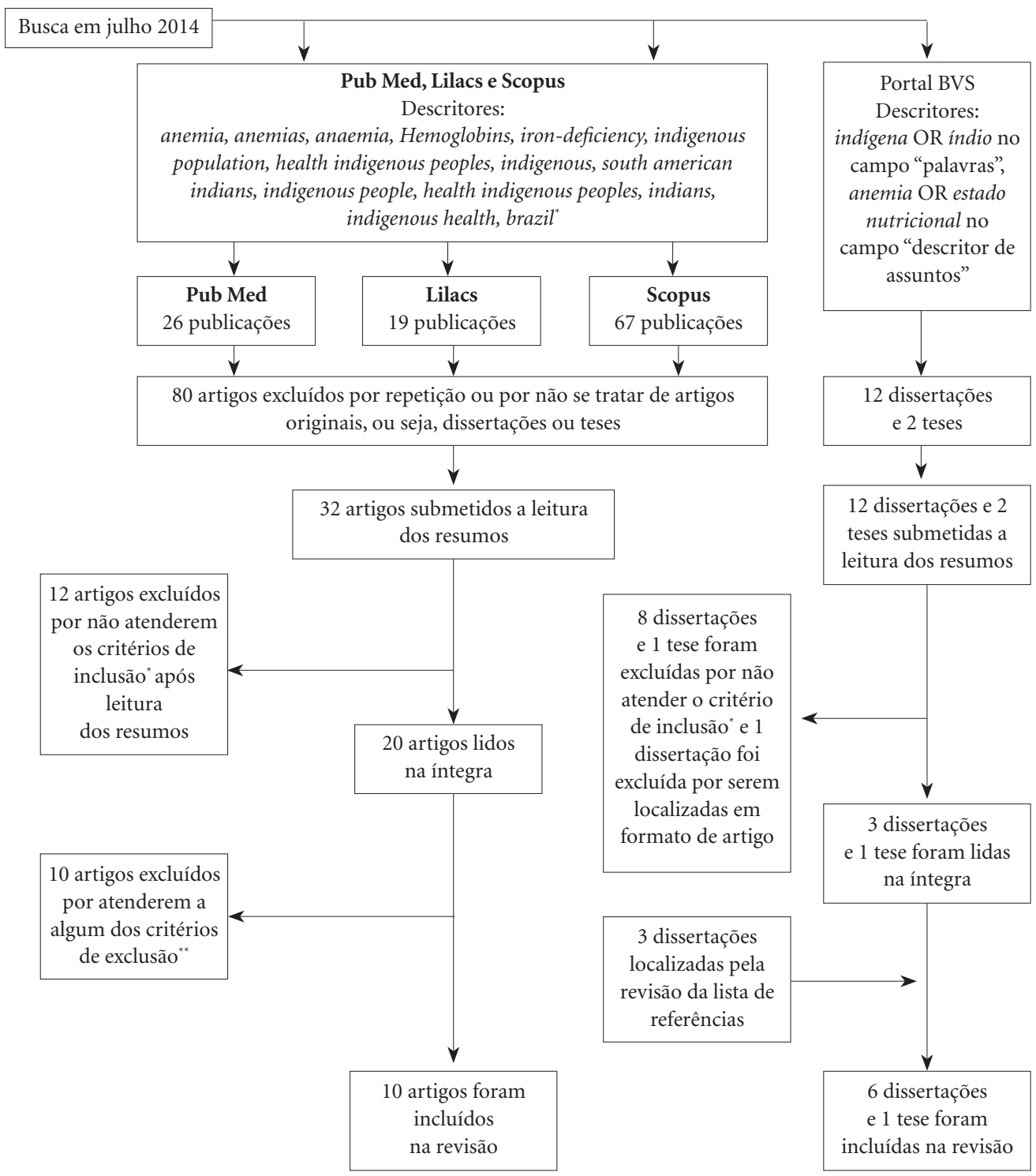

Figura 1. Fluxograma das fases da revisão sistemática.

"inclusão: estudos originais que tiveram como objetivo investigar a prevalência de anemia ferropriva e/ou fatores associados em crianças e mulheres indígenas no Brasil.

"* exclusão: identificação da anemia por método diferente da análise de hemoglobina e/ou ferro sério em análise laboratorial ou por hemoglobinômetro portátil; ausência da identificação precisa do local do estudo; não especificação do pertencimento étnico; artigos de revisão.

No Mato Grosso do Sul, entre as crianças Teréna da aldeia Limão Verde, os resultados reportados por Morais et al. ${ }^{20}$ demonstraram que $40,7 \%$ delas, na faixa etária dos seis aos 119 meses, estavam anêmicas, sendo maior a prevalência na faixa etária de seis aos 59 meses $(64,8 \%)$, sobretudo naquelas dos seis aos 23 meses $(86,1 \%)$. Pereira $^{13}$ investigou crianças Teréna das aldeias:
Barreirinho, Córrego do Meio e Lagoinha e encontrou prevalências menores em ambas as faixas de idade, 30,6\% crianças de seis aos 59 meses e 53,8\% para crianças de seis a 23 meses. Pereira ${ }^{13}$ foi o único estudo a utilizar o hemograma como método diagnóstico.

$\mathrm{O}$ estudo realizado por Mondini et al. ${ }^{23}$ encontrou elevada prevalência de anemia em crian- 
Tabela 1. Características dos estudos e da população estudada.

\begin{tabular}{|c|c|c|c|c|}
\hline Autor/Ano & Local/UF & Etnia & $\mathbf{N}$ & Faixa etária \\
\hline Coimbra Jr. e Santos $(1991)^{18}$ & Parque Indígena Arapuãna/RO & Suruí & 139 & $0-108$ meses \\
\hline Gugelmin $(1998)^{14}$ & Aldeia Pimentel Barbosa/MT & Xavante & 135 & Todas \\
\hline Serafim $(1997)^{21}$ & $\begin{array}{l}11 \text { aldeias no estado de São Paulo e } 3 \\
\text { aldeias no estado do Rio de Janeiro }\end{array}$ & Guarani & 178 & $0-60$ meses \\
\hline Leite $(1998)^{10}$ & Aldeia São José/MT & Xavante & 182 & $\begin{array}{r}0-60 \text { meses } \\
\text { Demais faixas }\end{array}$ \\
\hline Baruzzi et al. $(2001)^{24}$ & Aldeia Nacipotire/PA & Panará & 82 & $\begin{array}{r}6-59 \text { meses } \\
5-14 \text { anos }\end{array}$ \\
\hline Morais et al. $(2005)^{20}$ & $\begin{array}{l}\text { Aldeias: Limão Verde e Córrego Seco/ } \\
\text { MS }\end{array}$ & Teréna & 167 & $\begin{array}{r}6-72 \text { meses } \\
73-119 \text { meses }\end{array}$ \\
\hline Orellana et al. $(2006)^{19}$ & $\begin{array}{l}\text { Terra Indígena Sete de Setembro/RO/ } \\
\text { MT }\end{array}$ & Suruí & 268 & $\begin{array}{r}6-59 \text { meses } \\
60-119 \text { meses }\end{array}$ \\
\hline Mondini et al. $(2007)^{23}$ & Parque Indígena do Xingu/MT & Kamaiurá & 104 & $\begin{array}{r}6-59 \text { meses } \\
60-119 \text { meses }\end{array}$ \\
\hline Carvalho-Costa et al. $(2007)^{12}$ & Alto Rio Negro/AM & $\begin{array}{l}\text { Etnias do Alto } \\
\text { Rio Negro }\end{array}$ & 282 & $\begin{array}{r}6-59 \text { meses } \\
60-84 \text { meses }\end{array}$ \\
\hline Mondini et al. (2009) ${ }^{25}$ & Parque Indígena do Xingu/MT & Aruak e Karib ${ }^{c}$ & 269 & $\begin{array}{l}6-59 \text { meses } \\
60-119 \text { meses }\end{array}$ \\
\hline Koury Filho $(2010)^{15}$ & Município de Jordão/AC & Kaxinawá & 116 & mulheres* \\
\hline Orellana et al. $(2011)^{17}$ & $\begin{array}{l}\text { Terra Indígena Sete de Setembro/RO/ } \\
\text { MT }\end{array}$ & Suruí & 173 & $15-49,9$ anos \\
\hline Pereira $(2011)^{13}$ & $\begin{array}{l}\text { Aldeias: Barreirinho, Córrego do Meio } \\
\text { e Lagoinha/MS }\end{array}$ & Teréna & 49 & $0-59$ meses \\
\hline Souza $(2011)^{16}$ & $\begin{array}{l}\text { Aldeias: Pimentel Barbosa e } \\
\text { Etênhiritipá/MT }\end{array}$ & Xavante & 454 & $\begin{array}{r}6-59 \text { meses } \\
5-11 \text { anos } \\
12-14 \text { anos } \\
\text { Não gestantes } \geq \\
15 \text { anos }\end{array}$ \\
\hline Fávaro $(2011)^{11}$ & Terra Indígena Xukuru de Ororubá/PE & $\begin{array}{l}\text { Xukuru de } \\
\text { Ororubá }\end{array}$ & 1069 & $\begin{array}{r}6-59 \text { meses } \\
5-9 \text { anos } \\
\text { Não gestantes }< \\
15 \text { anos } \\
\text { Não gestantes } \geq \\
15 \text { anos }\end{array}$ \\
\hline Pereira et al. $(2012)^{26}$ & $\begin{array}{l}\text { Aldeia Plak-ô e povoado Terra Nova/ } \\
\text { AL }\end{array}$ & Karapotó & 99 & $6-59$ meses \\
\hline Barreto et al. $(2014)^{22}$ & $\begin{array}{l}\text { Aldeias: Sapucai, Parati Mirim, Sitio } \\
\text { Rio Pequeno, Araponga e Mamanguá/ } \\
\text { RS. Aldeia Boa Vista/SP }\end{array}$ & Guarani & 115 & $6-59$ meses \\
\hline
\end{tabular}

continua

ças indígenas Kamaiurá no Parque Indígena do Xingu, principalmente em crianças menores de 24 meses (81,3\%). Em outro estudo realizado também com crianças indígenas no Parque Indígena do $\mathrm{Xingu}^{25}$, foram encontradas elevadas taxas nas crianças Aruak e Karibe nesta mesma faixa etária.

Os estudos de Morais et al. ${ }^{20}$, Orellana et al..$^{19}$, Souza ${ }^{16}$, Fávaro ${ }^{11}$ e Pereira et al..$^{26}$ realizaram aná- lises a fim de identificar os fatores associados à presença de anemia em crianças. Leite ${ }^{10}$, por meio de regressão linear, investigou as variáveis associadas com a concentração de hemoglobina. Souza ${ }^{16}$ e Fávaro ${ }^{11}$ utilizaram a estimativa da Odds Ratio (OR) como medida de associação. Já os demais estudos ${ }^{19,20,26}$ fizeram a comparação de frequências (teste qui-quadrado ou exato de Fischer) para inferir associações. 


\begin{tabular}{|c|c|c|c|c|}
\hline Autor/Ano & & $\begin{array}{l}\text { diagnóstico } \\
\mathrm{g} / \mathrm{dl})\end{array}$ & $\begin{array}{c}\text { Recurso } \\
\text { diagnóstico }\end{array}$ & $\begin{array}{l}\text { Outras análises } \\
\text { realizadas no estudo }\end{array}$ \\
\hline Coimbra Jr. e Santos $(1991)^{18}$ & $<11,0$ & $(\mathrm{WHO}, 1975)^{27}$ & Método de Sahli & $\begin{array}{l}\text { Estado nutricional } \\
\text { Parasitismo intestinal }\end{array}$ \\
\hline Gugelmin $(1998)^{14}$ & $<11,0$ & $(\mathrm{WHO}, 1975)^{27}$ & $\mathrm{HemoCue}^{\circledR}$ & Estado nutricional \\
\hline Serafim $(1997)^{21}$ & $<11,0$ & $(\mathrm{WHO}, 1975)^{27}$ & HemoCue ${ }^{\circledR}$ & $\begin{array}{l}\text { Parasitismo intestinal } \\
\text { Consumo alimentar }\end{array}$ \\
\hline Leite $(1998)^{10}$ & $\begin{array}{l}<11,0 \\
<12,0\end{array}$ & $(\mathrm{WHO}, 1975)^{27}$ & HemoCue ${ }^{\circledR}$ & Estado nutricional \\
\hline Baruzzi et al. $(2001)^{24}$ & $\begin{array}{l}<11,0 \\
<12,0\end{array}$ & $(\mathrm{WHO}, 1989)^{28}$ & HemoCue ${ }^{\circledR}$ & $\begin{array}{l}\text { Estado nutricional } \\
\text { Tuberculose (bacilo de Koch) }\end{array}$ \\
\hline Morais et al. $(2005)^{20}$ & $\begin{array}{l}<11,0 \\
<11,5\end{array}$ & $(\mathrm{WHO}, 1999)^{29}$ & $\mathrm{HemoCue}^{\circledR}$ & Estado nutricional \\
\hline Orellana et al. $(2006)^{19}$ & $\begin{array}{l}<11,0 \\
<11,5\end{array}$ & $(\mathrm{WHO}, 2001)^{5}$ & $\mathrm{HemoCue}^{\circledR}$ & Estado nutricional \\
\hline Mondini et al. $(2007)^{23}$ & $\begin{array}{l}<11,0 \\
<11,5\end{array}$ & $(\mathrm{WHO}, 2001)^{5}$ & HemoCue $^{\circledR}$ & Estado nutricional \\
\hline Carvalho-Costa et al. $(2007)^{12}$ & $\begin{array}{l}<11,0 \\
<11,5\end{array}$ & $(\mathrm{WHO}, 2001)^{5}$ & $\mathrm{HemoCue}^{\circledR}$ & $\begin{array}{l}\text { Estado nutricional } \\
\text { Parasitismo intestinal }\end{array}$ \\
\hline Mondini et al. $(2009)^{25}$ & $\begin{array}{l}<11,0 \\
<11,5\end{array}$ & $(\mathrm{WHO}, 2001)^{5}$ & $\mathrm{HemoCue}^{\circledR}$ & Estado nutricional \\
\hline Koury Filho $(2010)^{15}$ & $<12,0$ & $(\mathrm{WHO}, 2001)^{5}$ & HemoCue ${ }^{\circledR}$ & $\begin{array}{l}\text { Estado nutricional } \\
\text { Indicadores socioeconômicos }\end{array}$ \\
\hline Orellana et al. $(2011)^{17}$ & $\begin{array}{l}\text { Não ges } \\
\text { Gestante }\end{array}$ & $\begin{array}{l}<12,0 \\
1,0(\mathrm{WHO}, 2001)^{5}\end{array}$ & $\mathrm{HemoCue}^{\circledR}$ & Indicadores socioeconômicos \\
\hline Pereira $(2011)^{13}$ & $<11,0$ & $(\mathrm{WHO}, 2001)^{5}$ & Hemograma $^{\mathrm{d}}$ & $\begin{array}{l}\text { Estado nutricional } \\
\text { Parasitismo intestinal }\end{array}$ \\
\hline Souza $(2011)^{16}$ & $\begin{array}{l}<11,0 \\
<11,5 \\
<12,0 \\
<12,0\end{array}$ & $(\mathrm{WHO}, 2001)^{5}$ & HemoCue ${ }^{\circledR}$ & $\begin{array}{l}\text { Estado nutricional } \\
\text { Indicadores socioeconômicos }\end{array}$ \\
\hline Fávaro $(2011)^{11}$ & $\begin{array}{l}<11,0 \\
<11,5 \\
<11,5 \\
<12,0\end{array}$ & $(\mathrm{WHO}, 2001)^{5}$ & HemoCue ${ }^{\circledR}$ & $\begin{array}{l}\text { Estado nutricional } \\
\text { Indicadores socioeconômicos }\end{array}$ \\
\hline Pereira et al. $(2012)^{26}$ & $<11,0$ & $(\mathrm{WHO}, 2001)^{5}$ & $\mathrm{HemoCue}^{\circledR}$ & $\begin{array}{l}\text { Estado nutricional } \\
\text { Indicadores socioeconômicos }\end{array}$ \\
\hline Barreto et al. $(2014)^{22}$ & $<11,0$ & $(\mathrm{WHO}, 2001)^{5}$ & $\mathrm{HemoCue}^{\circledR}$ & Estado nutricional \\
\hline
\end{tabular}

A investigação realizada entre as crianças Karapotó ${ }^{26}$, de Alagoas, identificou a associação da presença de anemia com a menor idade da criança (6-23 meses), menor tempo de estudo materno ( 5 anos ou menos de estudo), maior número de membros na família (mais de cinco integrantes), menor número de bens de consumo (cinco ou menos) e a moradia fora da aldeia (desaldeados). Para as crianças Xukuru do Ororubán ${ }^{11}$, de Pernambuco, e para as Teréna ${ }^{20}$, do Mato Grosso do Sul, a associação encontrada foi apenas em relação a menor idade, ou seja, aquelas com entre 6 e 23 meses foram as mais acometidas pelo $\operatorname{agravo}^{14,15}$.

Entre as crianças Xavante incluídas no estudo de Souza ${ }^{16}$, aquelas com idade entre 6 e 23 meses e as com irmãos onde o valor médio da hemoglobina foi menor de $11 \mathrm{~g} / \mathrm{dl}$ apresentaram chances, respectivamente, de 2,86 (IC95\%:1,15-7,12) e 10,70 (IC95\%:5,00-22,91) de serem anêmicas. 
Tabela 2. Prevalências de anemia em crianças e mulheres indígenas, segundo faixa de idade e sexo.

\begin{tabular}{|c|c|c|c|c|c|c|c|c|c|}
\hline \multirow[b]{3}{*}{ Autor/Ano } & \multicolumn{9}{|c|}{ Prevalência de anemia (\%) } \\
\hline & \multicolumn{3}{|c|}{ Crianças: 6 - 59 meses } & \multicolumn{3}{|c|}{ Crianças: 6 - 23 meses } & \multicolumn{3}{|c|}{ Crianças: 60 - 119 meses } \\
\hline & Mas & Fem & Total & Mas & Fem & Total & Mas & Fem & Total \\
\hline Coimbra Jr. e Santos (1991) $)^{18(a)}$ & & & $80,5 \%{ }^{(b)}$ & & & $78,8 \%$ & & & $59,8 \% 0^{(\mathrm{c})}$ \\
\hline \multicolumn{10}{|l|}{ Gugelmin $(1998)^{14}$} \\
\hline Serafim $(1997)^{21(d)}$ & & & $68,5 \%$ & $89,3 \%$ & $75,7 \%$ & $81,5 \%$ & & & \\
\hline Leite $(1998)^{10}$ & $73,2 \%$ & $75,8 \%$ & $74,3 \%$ & & & $96,8 \%$ & $79,3 \%$ & $66,7 \%$ & $73,2 \%$ \\
\hline Baruzzi et al. $(2001)^{24}$ & & & $33,3 \%{ }^{(\mathrm{g})}$ & & & & & & $73,9 \% 0^{(\mathrm{h})}$ \\
\hline Morais et al. $(2005)^{20}$ & & & $64,8 \%$ & & & $86,1 \%$ & & & $40,7 \%$ \\
\hline Orellana et al. $(2006)^{19(\mathrm{i})}$ & $84,7 \%$ & $83,3 \%$ & $84,0 \%$ & $92,0 \%$ & $92,6 \%$ & $92,3 \%$ & $74,0 \%$ & $80,8 \%$ & $76,6 \%$ \\
\hline Mondini et al. $(2007)^{23}$ & & & $60,0 \%$ & & & $81,3 \%$ & & & $50,0 \%$ \\
\hline Carvalho-Costa et al. $(2007)^{12}$ & & & & & & & & & $51,1 \%^{(j)}$ \\
\hline \multicolumn{10}{|l|}{ Mondini et al. $(2009)^{25}$} \\
\hline Aruak & & & & & & $83,3 \%$ & & & $61,5 \%$ \\
\hline Karibe & & & & & & $84,6 \%$ & & & $67,3 \%$ \\
\hline \multicolumn{10}{|l|}{ Koury Filho $(2010)^{15}$} \\
\hline \multicolumn{10}{|l|}{ Orellana et al. $(2011)^{17}$} \\
\hline Pereira $(2011)^{13}$ & & & $30,6 \%$ & & & $53,8 \%$ & & & \\
\hline Souza $(2011)^{16}$ & $62,1 \%$ & $55,3 \%$ & $58,5 \%$ & & & & $42,4 \%$ & $51,8 \%$ & $47,0 \%$ \\
\hline Fávaro $(2011)^{11}$ & $31,4 \%$ & $27,9 \%$ & $29,6 \%$ & $40,5 \%$ & $45,5 \%$ & $42,9 \%$ & $18,7 \%$ & $15,1 \%$ & $17,0 \%$ \\
\hline Pereira et al. $(2012)^{26}$ & $56,5 \%$ & $58,4 \%$ & $57,6 \%$ & & & $72,7 \%$ & & & \\
\hline Barreto et al. $(2014)^{22}$ & $73,3 \%$ & $57,4 \%$ & $65,2 \%$ & $71,4 \%$ & $80,0 \%$ & $76,4 \%$ & & & \\
\hline
\end{tabular}

\begin{tabular}{|c|c|c|c|c|}
\hline \multirow[b]{3}{*}{ Autor/Ano } & \multicolumn{4}{|c|}{ Prevalência de anemia (\%) } \\
\hline & \multicolumn{3}{|c|}{ Crianças: 6 - 119 meses } & \multirow{2}{*}{ Mulheres } \\
\hline & Mas & Fem & Total & \\
\hline Coimbra Jr. e Santos $(1991)^{18(a)}$ & $65,4 \%$ & $78,7 \%$ & $71,2 \%$ & \\
\hline Gugelmin $(1998)^{11}$ & $40,0 \%$ & $44,4 \%$ & $42,1 \%$ & $\begin{array}{l}10-20 \text { anos: } 17,6 \% \\
20-40 \text { anos: } 18,8 \% \\
\geq 40 \text { anos: } 29,4 \%\end{array}$ \\
\hline \multicolumn{5}{|l|}{ Serafim $(1997)^{21(d)}$} \\
\hline Leite $(1998)^{10}$ & & & & $\begin{array}{l}15-40 \operatorname{anos}(e): 52,9 \% \\
15-40 \operatorname{anos}(f): 29,4 \%\end{array}$ \\
\hline Baruzzi et al. $(2001)^{24}$ & & & $42,0 \%$ & \\
\hline Morais et al. $(2005)^{20}$ & & & $62,3 \%$ & \\
\hline Orellana et al. $(2006)^{19(i)}$ & $79,2 \%$ & $82,3 \%$ & $80,6 \%$ & \\
\hline Mondini et al. $(2007)^{23}$ & $64,4 \%$ & $47,5 \%$ & $55,3 \%$ & \\
\hline \multicolumn{5}{|l|}{ Carvalho-Costa et al. $(2007)^{12}$} \\
\hline \multicolumn{5}{|l|}{ Mondini et al. (2009) ${ }^{25}$} \\
\hline Aruak & $65,9 \%$ & $72,2 \%$ & $66,0 \%$ & \\
\hline Karibe & $70,1 \%$ & $70,0 \%$ & $74,6 \%$ & \\
\hline Koury Filho $(2010)^{15}$ & & & & $44,1 \%{ }^{(\mathrm{k})}$ \\
\hline Orellana et al. $(2011)^{17}$ & & & & $\begin{array}{l}15-49 \text { anos gestantes: } 81,8 \% \\
15-49 \text { anos: } 67,3 \%\end{array}$ \\
\hline \multicolumn{5}{|l|}{ Pereira $(2011)^{13}$} \\
\hline Souza $(2011)^{16}$ & & & & $\begin{array}{l}12-15 \text { anos: } 46,7 \% \\
15-18 \text { anos: } 65 \% \\
18-40 \text { anos: } 55,1 \% \\
\geq 40 \text { anos: } 51,4 \%\end{array}$ \\
\hline $\begin{array}{l}\text { Fávaro }(2011)^{11} \\
\text { Pereira et al. }(2012)^{26} \\
\text { Barreto et al. }(2014)^{22}\end{array}$ & $24,4 \%$ & $21,7 \%$ & $23,1 \%$ & $10-49$ anos: $16,1 \%$ \\
\hline
\end{tabular}

(a) Neste estudo foram incluídas crianças de até 9 anos ou 108 meses. $\mathrm{O}$ artigo original apresentava as prevalências fracionadas por ano, que foram recalculadas para se ajustarem à tabela. ${ }^{(b)}$ Neste estudo também foram incluídas crianças com idade entre 0 e 6 meses. ${ }^{(c)}$ Neste estudo não foram incluídas crianças com idade entre 108 e 119 meses. ${ }^{(d)}$ Neste estudo foram consideradas as crianças de 0 a 65 meses. ${ }^{(e)}$ Critério diagnóstico: $<12 \mathrm{~g} / \mathrm{dl} .{ }^{(\mathrm{ff}}$ Critério diagnóstico: $<11 \mathrm{~g} / \mathrm{dl} .{ }^{(\mathrm{g})}$ Esta prevalência é referente a crianças Panará com idade de 6 a 71 meses. ${ }^{(\mathrm{h})}$ Esta prevalência é referente a crianças Panará com idade de 72 a 168 meses. ${ }^{(i)}$ Neste estudo as prevalências pelas faixas etárias indicadas foram recalculadas, uma vez que o artigo original apresentava as prevalências com outros agrupamentos etários, cujos resultados estavam detalhados e permitiram tal ajuste. ${ }^{(j)}$ Esta prevalência é referente a crianças do Alto Rio Negro com idade de 6 a 84 meses. ${ }^{(k)}$ Não informou a idade das mulheres. 
Orellana et al. ${ }^{19}$ não encontraram associação entre as variáveis investigadas e a presença de anemia em crianças Suruí, de Rondônia e Mato Grosso. Leite ${ }^{10}$ observou, para as crianças Xavantes menores de 10 anos, a tendência de crescimento da concentração de hemoglobina com o aumento da idade, além de uma associação estatisticamente significante entre as dosagens desta com os valores de z-escore do índice antropométrico massa corporal-para-estatura.

Em dois estudos realizados com mulheres, Suruí $^{17}$ e Xukuru do Ororubá ${ }^{11}$, foram investigados os fatores associados à anemia, sendo que ambos utilizaram a Odds Ratio (OR) como medida de associação, estimada através de regressão logística múltipla. No estudo com as mulheres Suruí ${ }^{17}$ observou-se que aquelas com um ou dois filhos anêmicos com idade entre 6 e 35 meses tiveram 3,0 (IC95\%: 1,3-7,0) vezes mais chance de serem anêmicas, assim como as mulheres em extratos socioeconômicos mais baixos $(\mathrm{OR}=3,5$; IC95\%: 1,3-9,3). Entre as mulheres Xukuru ${ }^{11}$, a única variável associada à presença de anemia, após o ajuste por uma série de variáveis socioeconômicas e demográficas, foi a escolaridade, em que mulheres com oito ou mais anos de estudo apresentaram chance 2,2 (IC95\%: 1,12-4,43) menores de apresentarem anemia quando comparadas às com menos escolaridade.

\section{Discussão}

As prevalências de anemia reportadas nos estudos incluídos nesta revisão se alinham às observadas no Inquérito Indígena e confirmam a gravidade da situação neste segmento populacional, com prevalências extremamente elevadas, principalmente entre as crianças. De acordo com a $\mathrm{WHO}^{5}$, as prevalências superiores a $40 \%$ colocam a anemia como grave problema de saúde pública entre povos indígenas no Brasil.

Considerada um indicador de situações precárias de saúde e nutrição ${ }^{5}$, a anemia é associada ao risco aumentado de mortalidade infantil e materna, a consequências negativas para o desenvolvimento físico e cognitivo de crianças e à baixa produtividade de adultos ${ }^{2}$. Leal e Osório ${ }^{4}$ realizaram uma revisão sistemática a fim de investigar os principais fatores associados à presença de anemia nas crianças e argumentam que idade, baixa escolaridade materna, baixo nível socioeconômico, déficit de peso e estatura em relação ao padrão, diarreia, baixa densidade de ferro na dieta e densidade de calorias provenientes do leite de vaca são os fatores mais frequentemente associados a anemia.

A nível nacional, o primeiro inquérito a medir a prevalência de anemia em crianças menores de 5 anos e em mulheres de 15 a 49 anos não indígenas (PNDS/2006-2007) ${ }^{30}$ mostrou resultados mais favoráveis aos que vinham sendo apresentados em estudos pontuais realizados em diversas regiões do Brasil ${ }^{4}$. Foram encontradas prevalências de $20,9 \%$ para crianças menores de cinco anos e de $29,4 \%$ para mulheres entre 15 e 49 anos. Vieira e Ferreira ${ }^{3}$ alertam para as diferenças metodológicas no tocante à quantificação do nível de hemoglobina entre a PNDS e a maioria dos estudos pontuais realizados no Brasil. Enquanto grande parte dos estudos utilizou o Hemocue, a PNDS/2006-2007 fez uso de amostras de sangue depositadas em papel filtro para análise através do método da cianometa-hemoglobina ${ }^{29}$. Tal diferença no método de identificação do agravo talvez possa justificar as diferenças observadas, mas ainda assim, as prevalências de anemia obtidas na PNDS também foram extremamente elevadas.

Em todos os estudos identificados nesta revisão, com exceção o de Coimbra Júnior e Santos ${ }^{18}$ e o de Pereira ${ }^{13}$, a concentração da hemoglobina em $\mathrm{g} / \mathrm{dl}$ foi obtida através da leitura direta em fotômetro portátil (hemoglobinômetro HemoCue) por meio de amostra capilar de polpa digital. A concentração de hemoglobina que identificou a presença de anemia em crianças menores de cinco anos foi similar em todos os estudos (< $11,0 \mathrm{~g} / \mathrm{dl}$ ). Ainda é importante citar que o Inquérito Indígena também utilizou igual metodologia e ponto de corte para a identificação da anemia em menores de cinco anos. Tais constatações reforçam a pertinência quanto à comparação entre a grande maioria dos estudos identificados.

A investigação sobre o perfil nutricional em crianças indígenas Suruí realizada há mais de 25 anos constatou elevada prevalência de anemia, baixo peso para a idade, déficit de crescimento e infestações parasitárias ${ }^{18}$. Este quadro foi atribuído à falta de tratamento da água, aos dejetos, à remoção de lixo e à alimentação inadequada, resultantes da redução da capacidade de produção de alimentos tradicionais, damonotonia alimentar e do consumo de alimentos industrializados. Após duas décadas, o perfil nutricional de crianças Suruí foi novamente investigado ${ }^{19} \mathrm{e}$, apesar de uma sutil diminuição da desnutrição, a prevalência de anemia manteve-se inalterada. Destaca-se que tal comparação merece cautela, uma vez que os métodos e os critérios diagnósticos foram distintos. Os autores argumentaram que as incrementadas 
taxas de anemia observadas, associadas ao déficit de crescimento, refletiam a manutenção das precárias condições sanitárias e de insegurança alimentar.

$\mathrm{Na}$ mesma época do estudo de Orellana et al. ${ }^{19}$, também foi observada em crianças Kamaiurá, do Alto Xingu, alta prevalência de anemia ${ }^{24}$ Neste caso, a presença acentuada de anemia foi atribuída à elevada proporção de infecções parasitárias que acometiam a localidade. Para os autores, o aleitamento materno até os dois ou três anos somado à alimentação complementar inserida somente ao final do primeiro ano de vida e composta basicamente por peixe, mingau de mandioca e beiju umedecido, pode ter favorecido a elevada prevalência $(81,3 \%)$ de anemia nas crianças Kamaiurá menores de 24 meses.

Ainda que elevada, a menor prevalência de anemia $(29,6 \%)$ em crianças indígenas com menos de cinco anos foi identificada entre as Xukuru do Ororubá ${ }^{11}$, de Pernambuco. Os resultados do Inquérito Indígena para a região Nordeste também registraram as menores prevalências (40,9\%) em comparação às demais regiões do Brasil. Por outro lado, as prevalências reportadas da PNDS/2006-2007 mostraram que nesta região estavam concentradas as maiores prevalências de anemia em mulheres e crianças não indígenas, $39,1 \%$ e $25,5 \%$, respectivamente. Os povos indígenas da região Nordeste representam uma parcela expressiva do contingente indígena no país, porém ainda são escassas as publicações que abordem as questões de saúde e nutrição destes povos.

Os estudos que também realizaram a avaliação antropométrica indicaram elevadas prevalências de desnutrição, especialmente no que tange ao déficit de crescimento. As situações mais favoráveis foram observadas entre as crianças Panará, Xukuru e Teréna, embora não tenham interferido nos resultados desfavoráveis quanto a anemia nestes povos ${ }^{11,20,23}$.

A avaliação da saúde realizada com os índios Panará, que vivem no sul do Pará, encontrou uma situação mais favorável no que diz respeito à anemia, quando comparada a de outros povos indígenas ${ }^{23}$. Os valores reportados indicam que a prevalência de anemia foi inferior nas crianças menores de cinco anos quando comparadas às maiores, com idade entre 5 e 14 anos (33,3\% $\$ 73,9 \%)$. Sendo importante destacar a diferença deste com a maioria dos estudos realizados em contextos indígenas e não indígenas, que reportam prevalências superiores em crianças menores de cinco anos ${ }^{3,8}$. Este estudo com os índios Panará poderia encontrar prevalências ainda mais elevadas nas crianças maiores, uma vez que o ponto de corte utilizado para a definição de anemia foi de hemoglobina inferior a $12 \mathrm{~g} / \mathrm{dl}$, e nos dos demais estudos a anemia apenas foi diagnostica com valores menores de $11,5 \mathrm{~g} / \mathrm{dl}$, principalmente nas crianças entre 5 e 9 anos.

Entre as mulheres indígenas, considerando todas as faixas de idade incluídas nos estudos, o perfil de anemia observado também coloca este seguimento populacional em uma situação vulnerável, a exemplo dos estudos com as Suruí. Nestas, foram observadas prevalências globais elevadas para gestantes $(67,3 \%)$ e não gestantes $(81,8 \%)^{14,17}$. De fato, as mulheres, durante seu período reprodutivo, possuem uma demanda alta por nutrientes hematopoiéticos e, quando não estão grávidas ou lactantes, as perdas menstruais regulares constituem uma depleção constante de nutrientes que devem ser repostos. Esses fatores, juntamente com a carência dietética e a dificuldade de absorção, podem levar a um desequilíbrio, ocasionando deficiência de ferro e anemia ${ }^{2}$.

Os determinantes dos agravos nutricionais nos indígenas estão relacionados a inúmeras transformações de ordem demográfica, socioeconômica, cultural, ambiental e no seu perfil epidemiológico. Em muitas comunidades indígenas, a subsistência a partir de atividades de caça, coleta e pesca vêm ao longo dos anos passando por transformações decorrentes principalmente da instalação de novos regimes econômicos e da diminuição dos limites territoriais, levando frequentemente a uma situação de empobrecimento da dieta ${ }^{7}$.

É notório que a partir de 2001 houve um aumento significativo de estudos que investigaram a ocorrência de anemia nas populações indígenas no país. Possivelmente este aumento está associado à inserção da área de saúde indígena no campo da saúde pública/coletiva no país, passando a ocupar um espaço importante no plano dos debates sobre a situação de saúde e as desigualdades sociais.

Como foi detalhado, os trabalhos analisados constituíram-se, basicamente, em estudos de caso, enfocando etnias específicas, avaliadas em contextos temporais bem delimitados. A consolidação dos sistemas de informação da saúde indígena somados às pesquisas acadêmicas, como as analisadas neste trabalho, e a outras iniciativas, como o já referido Inquérito Indígena ${ }^{9}$, pode produzir informações que melhor caracterizem a situação nutricional dos povos indígenas. No entanto, para além da caracterização da situação de 
saúde dos povos indígenas no Brasil, que já está dada e é gravíssima, é fundamental que tais informações, de fato, contribuam para gerar prioridades de atenção à saúde nestas comunidades.

\section{Colaboradores}

JSA Lício e TR Fávaro participaram igualmente de todas as etapas de produção do artigo: concepção, análise e interpretação dos dados, redação, revisão crítica do conteúdo e aprovação final da versão a ser publicada. CRMM Chaves contribuiu com a concepção, a interpretação dos dados, a redação e a revisão crítica do conteúdo.

\section{Agradecimentos}

Este trabalho foi realizado durante a vigência bolsa de pós-doutorado (Convênio Fiocruz/ Capes - Brasil sem Miséria) concedida a segunda autora do artigo (TR Fávaro). 


\section{Referências}

1. Saloojee H, Pettifor JM. Iron deficiency and impaired child development. BMJ 2001; 323(7326):1377-1378.

2. World Health Organization (WHO). Worldwide prevalence of anaemia 1993-2005: WHO global database on anemia. Geneva: WHO; 2008.

3. Vieira RCS, Ferreira HS. Prevalência de anemia em crianças brasileiras, segundo diferentes cenários epidemiológicos. Rev. Nutr. 2010; 23(3):433-444.

4. Leal LP, Osório MM. Fatores associados à ocorrência de anemia em crianças menores de seis anos: uma revisão sistemática dos estudos populacionais. Rev Bras Saúde Mater Infant 2010; 10(4):417-439.

5. World Health Organization (WHO). Iron Deficiency Anaemia Assessment, Prevention, and Control: A guide for programme managers. Geneva: WHO; 2001.

6. Khambalia AZ, Ashley MA, Stanley HZ. Burden of anemia among indigenous populations. Nutrition Reviews 2011; 69(12):693-719.

7. Horta BL, Santos RV, Welch JR, Cardoso AM, dos Santos JV, Assis AM, Lira PC, Coimbra Júnior CEA. Nutricional status of indigenous children: findings from the First National Survey of Indigenous People's Health and Nutrition in Brazil. Int J Equity Health 2013; 12:23.

8. Coimbra Júnior CEA, Santos RV, Welch JR, Cardoso AM, Souza MC, Garnelo L, Rassi E, Follér ML, Horta BL. The First National Survey of Indigenous People's Health and Nutrition in Brazil: rationale, methodology, and overview of results. BMC Public Health 2013; 13:52.

9. Leite MS, Cardoso AM, Coimbra Jr CEA, Welch J, Gugelmin AS, Lira PC, Horta BL, Santos RV, Escobar AL. Prevalence of anemia an associated factors among indigenous children in Brazil: results from the First National Survey of Indigenous People's Health and Nutrition. Nutr J 2013; 12-69.

10. Leite MS. Avaliação do estado nutricional da população Xavante de São José, Terra Indígena Sangradouro - Volta Grande, Mato Grosso [dissertação]. Rio de Janeiro: Fundação Oswaldo Cruz; 1998.

11. Fávaro TR. Perfil nutricional da população indígena Xukuru do Ororubá, Pernambuco, Brasil [tese]. Rio de Janeiro: Fundação Oswaldo Cruz; 2011.

12. Carvalho-Costa FA, Gonçalves AQ, Lassance SL, Silva Neto LM, Salmazo CAA. Giardia Lamblia and other intestinal parasitic infections and their relationships with nutritional status in children in Brazilian amazon. Rev Inst Med Trop S Paulo 2007; 49(3):147-153.

13. Pereira AO. Prevalência de anemia por deficiência de ferro em crianças indígenas Terena [dissertação]. Campo Grande: Universidade Federal do Mato Grosso do Sul; 2011.

14. Gugelmin SA. Nutrição e alocação de tempo dos Xavantes de Pimentel Barbosa, Mato Grosso: um estudo em ecologia humana e mudanças [dissertação]. Rio de Janeiro: Fundação Oswaldo Cruz; 1998.

15. Koury Filho HC. Estado nutricional de mulheres, mães de crianças menores de cinco anos [dissertação]. São Paulo: Universidade de São Paulo; 2010.

16. Souza JAM. Prevalência de anemia e fatores associados nas aldeias Xavante de Pimentel Barbosa e Etênhiritipá, Mato Grosso, Brasil [dissertação]. Rio de Janeiro: Fundação Oswaldo Cruz; 2011.
17. Orellana JDY, Cunha MG, Santos RV, Coimbra Júnior CEA, Leite MS. Prevalência e fatores associados à anemia em mulheres indígenas Suruí com idade de 15 a 49 anos, Amazônia, Brasil. Rev Bras Saúde Matern Infant 2011; 11(2):153-161.

18. Coimbra Júnior CEA, Santos RV. Avaliação do estado nutricional num contexto de mudança sócio-econômica: o grupo indígena Suruí do Estado de Rondônia, Brasil. Cad Saude Publica 1991; 7(4):538-562.

19. Orellana JDY, Coimbra Júnior CEA, Lourenço AEP, Santos RV. Estado nutricional e anemia em crianças indígenas Suruí, Amazônia, Brasil. J Pediatr 2006; 82(5):383388.

20. Morais MB, Alves GMS, Fagundes Neto U. Estado nutricional de crianças índias terenas: evolução do peso e estatura e prevalência atual de anemia. J Pediatr 2005; 81(5):383-389.

21. Serafim MG. Hábitos alimentares e nível de hemoglobina em crianças indígenas Guarani, menores de cinco anos, dos estados de São Paulo e do Rio de Janeiro [dissertação]. São Paulo: Universidade Federal de São Paulo; 1997.

22. Barreto CTG, Cardoso AM, Coimbra Júnior CEA. Estado nutricional de crianças indígenas guarani nos estados de Rio de Janeiro e São Paulo. Cad Saude Publica 2014; 30(3):657-662.

23. Mondini L, Canó EN, Fagundes U, Lima EES, Rodrigues D, Baruzzi RG. Condições de nutrição em crianças Kamaiurá-povo indígena do Alto Xingu, Brasil Central. Rev Bras Epidemiol 2007; 10(1):39-47.

24. Baruzzi RG, Barros VL, Rodrigues D, Souza ALM, Pagliaro H. Saúde e doença em índios Panará (Kreen -Akarôre) após vinte e cinco anos de contato com o nosso mundo, com ênfase na ocorrência de tuberculose (Brasil Central). Cad Saude Publica 2001; 17(2):407412.

25. Mondini L, Rodrigues DA, Gimeno SGA, Baruzzi RG. Estado Nutricional e níveis de hemoglobina em crianças Aruak e Karibe- povos indígenas do Alto Xingu, Brasil Central, 2001-2002. Rev Bras Epidemiol 2009; 12(3):469-477.

26. Pereira JF, Oliveira MAO, Oliveira JS. Anemia em crianças indígenas da etnia Karapotó. Cad Saude Publica 2012; 12(4):375-382.

27. World Health Organization (WHO). Control of Nutritional anaemia with special reference to iron deficiency. Geneva: WHO; 1975. Technical Report Series 580.

28. World Health Organization (WHO). Preventing and controlling anaemia through primary health care: a guide for health administrators and programme managers. Geneva: WHO; 1985

29. World Health Organization (WHO). Prevention and Control of Iron Deficiency Anaemia in Women and Children. Geneva: WHO; 1999.

30. Brasil. Ministério da Saúde (MS). Centro Brasileiro de Análise e Planejamento. Pesquisa Nacional de Demografia e Saúde da Criança e da Mulher - PNDS 2006: dimensões do Processo Reprodutivo e da Saúde da Criança. Brasília: MS; 2009.

Artigo apresentado em 25/09/2014

Aprovado em 16/06/2015

Versão final apresentada em 18/06/2015 
